\title{
Le syndrome hollandais à l'épreuve des faits au Gabon
}

\author{
Jacques Janvier Rop's Okoué Edou \\ Faculté des sciences de l'administration \\ Université du Québec à Rimouski
}

\section{INTRODUCTION}

«Like New-York's psychiatrits, norways economists find themselves specialising in the diseases of the rich ${ }^{1}$, voici en quels termes la revue The Economist choisit de commencer, dans son numéro du 18 avril 1981, un article consacré aux problèmes posés à la Norvège par les revenus tirés du pétrole. Quelques années plutôt, cette revue avait présenté le cas des Pays-Bas et décrit les conséquences que les exportations massives de gaz naturel avaient entraînées sur son économie, proposant pour les désigner l'expression, désormais célèbre, de « dutch disease » ou «maladie néerlandaise ». Cette dernière est apparue vers $1975^{2}$, au moment où eurent lieu les débats relatifs aux problèmes que le pétrole de la mer du Nord risquait de poser à la Grande-Bretagne. Elle fait référence aux difficultés rencontrées par la Hollande à la suite de l'exploitation, dans les années 1970, des réserves de gaz naturel du gisement Slochteren. Cette référence à une certaine morbidité associée à l'exploitation d'une ressource naturelle peut surprendre et paraitre paradoxale au sens où les expériences de la plupart des pays pétroliers et miniers révèlent que la possession des richesses naturelles n'a pas toujours l'effet favorable escompté sur l'évolution économique. La possession ou l'exploitation d'une ressource naturelle déclenche un processus d'ajustement aboutissant généralement au déclin des branches exposées à la concurrence internationale et à l'expansion des branches qui en sont abritées. Un boom (pétrolier ou minier) tendrait donc spontanément à compromettre tout projet d'industrialisation ou de diversification des exports, aggravant ainsi la vulnérabilité de l'économie.

Toutefois, le «mal néerlandais » n'est pas l'apanage des pays pétroliers ou miniers. Ses symptômes ont été également identifiés dans des économies affectées par des chocs aussi différents que le développement rapide de secteurs à haute technologie et dans les pays à dominante agricole lorsqu'ils connaissent une brusque valorisation de leurs produits primaires agricoles. Dès lors, dans une première acception, le terme de «dutch disease » peut être assimilé à la «pétrolisation» dans le cas où la ressource naturelle serait le pétrole. Il s'agit alors de démontrer le caractère excluant des exports du pétrole vis-à-vis des autres produits d'exports traditionnels. Cependant, comme cette maladie ne résulte pas uniquement de l'exportation du pétrole, mais également de l'exportation d'autres produits de base (cacao, café, cuivre), elle désigne alors l'ensemble des effets préjudiciables créés dans une économie par l'expansion du secteur qui produit une ressource naturelle. Selon Daniel ${ }^{3}, \ll$ il se traduit par de brusques modifications dans l'attribution des ressources, par des changements de la structure sectorielle du système productif, les mouvements des prix relatifs étant au centre de ces distorsions sectorielles ».

\section{Elle fait référence aux difficultés rencontrées par la Hollande à la suite de l'exploitation, dans les années 1970, des réserves de gaz naturel du gisement Slochteren.}

Relativement au Gabon, c'est précisément un choc de ce type qu'il subit depuis la décennie 1980 à la suite de la flambée des cours internationaux du brut. Après la brusque montée des prix des produits pétroliers du début des années 1970, ce pays a en effet connu, à l'instar des autres pays producteurs de pétrole, les effets bénéfiques du transfert de revenus (rentrées de devises) en provenance des pays acheteurs. Cependant, cet effet d'enrichissement global du Gabon à la suite de la hausse du cours de l'or noir s'est très vite paradoxalement accompagné d'effets sectoriels pervers se traduisant par un déclin des branches exposées à la concurrence internationale hors boom comme le bois, les mines (manganèse et uranium), l'agriculture. Devant le 
dépérissement de la filière agricole, par exemple, la demande interne stimulée par l'augmentation du revenu réel s'est tournée vers les biens étrangers similaires plus compétitifs, provoquant au passage une explosion des importations et une dégradation du solde extérieur en biens alimentaires. L'ensemble de ces effets préjudiciables créés au sein de l'économie par le boom pétrolier nous pousse à dire que le Gabon connaît un «syndrome néerlandais ».

Dans ce cadre, le sujet d'étude faisant l'objet de la recherche scientifique ici est «le syndrome hollandais à l'épreuve des faits au Gabon ». Le cadre référentiel choisi permet de mettre en exergue la théorie du «syndrome hollandais » et les concepts sous-jacents (effet de déplacement des ressources productives; effet de dépense), à l'aulne la politique pétrolière menée par les autorités publiques dans les années 1970, du boom du pétrole et du leadership de ce produit dans les principaux agrégats économiques dès les années 1980 . Sur la base du cadre conceptuel susmentionné, il s'agit de savoir si le

\section{LA THÉORIE DU SYNDROME HOLLANDAIS}

\subsection{Le modèle originel de R.G. Grégory}

Selon R.G. Grégory, «le développement des ressources naturelles implique un nécessaire déclin relatif des autres secteurs de l'économie $»^{7}$. Ce dernier mit en lumière les changements structurels prévisibles en Australie à la suite de l'expansion du secteur minier. Il élabora un modèle exposant les effets des prix domestiques sur l'offre d'exports et la demande d'imports. Par ailleurs, il étudia le rôle du taux de change réel (TCR) dans les effets d'un boom sur les secteurs d'exports et d'imports.

Dans ce contexte, il nota que le développement de toute ressource naturelle destinée à l'exportation (pétrole, gaz, bois, mines, cacao, café) ou au remplacement des importations affecte la balance commerciale et conduit, au travers de l'appréciation du taux de change réel, au déclin relatif de la production domestique des biens exposés (trade sector) hors boom.

Le développement de toute ressource naturelle destinée à l'exportation (pétrole, gaz, bois, mines, cacao, café) ou au remplacement des importations affecte la balance commerciale. boom du pétrole issu de la politique pétrolière de la décennie 1970 a, au travers des effets de «déplacement des ressources productives » et de « dépenses », engendré une domination du produit «pétrole » dans le produit intérieur brut $(P I B)$, les recettes d'exportations et les recettes budgétaires. L'étude statistique des effets de « déplacement des ressources productives » et de « dépenses » s'inscrit dans une approche théorique «pluraliste $»^{4}$ et ce, sous le paradigme du «positivisme ». Le devis ${ }^{5}$ de la présente recherche scientifique se veut essentiellement explicatif au sens de simplement établir une relation d'asymétrie temporelle ${ }^{6}$ entre le boom du pétrole issu de la politique pétrolière de la décennie 1970 et la modification de la structure de l'économie gabonaise au profit (détriment) du pétrole (hors pétrole) dès les années 1980 . Il repose principalement sur deux techniques de collecte de données (analyse du contenu et analyse des statistiques) et ce, sur une approche par variables (valeur ajoutée pétrolière; main-d'œuvre agricole; recettes d'exportations pétrolières).

\subsection{Le modèle de référence de W.M. Corden et J.P. Neary}

La pathologie du «dutch disease » initiée par R.G. Gregory a été approfondie par W.M. Corden et J.P. Neary ${ }^{8}$. Dans une étude publiée en 1982, ces deux derniers élaborèrent un modèle destiné à appréhender l'impact du «mal néerlandais » à l'aulne d'un certain nombre d'hypothèses.

H1 : L'économie considérée est censée être de petite dimension.

C'est une petite économie ouverte produisant deux biens commercialisés (internationalement) dont les prix (exogènes) sont donnés et un bien non commercialisé (internationalement) dont le prix flexible s'établit par confrontation de l'offre et de la demande domestiques. Cette petite économie comprend donc trois secteurs (celui exposé, Sc, où se produit le choc positif; celui exposé, Se, regroupant l'ensemble des autres biens échangeables; celui non exposé, Sne).

H2 : L'analyse se concentre sur le court terme. 
Chaque secteur a un facteur spécifique (le capital) et un facteur mobile (le travail). Toutefois, seul le travail circule librement dans toute l'économie, le capital étant spécifique à chaque secteur.

H3 : Tous les biens sont utilisés au départ pour la consommation finale.

H4 : Le modèle fait fi des facteurs monétaires et tient seulement compte des prix relatifs.

Dans ce contexte, W.M. Corden et J.P. Neary se proposèrent d'étudier l'impact d'un boom dans Sc sur le secteur des autres biens échangeables hors boom $(\mathrm{Se})$ et le secteur des biens non échangeables (Sne). Selon ces deux auteurs, l'impact de l'expansion du secteur en boom $(S c)$ peut être ramené à deux effets distincts. D'un côté, il s'agit d'un effet de réallocation des ressources productives (ressource movement effect) qui est relatif au déplacement du facteur mobile $(L)$ des secteurs des biens abrités ( $S n e$ ) et des autres biens échangeables hors boom $(\mathrm{Se})$ vers le secteur en boom $(\mathrm{Sc})$. De l'autre, il s'agit d'un effet de dépense (spending effect) qui a, quant à lui, trait à l'utilisation des revenus issus du boom.

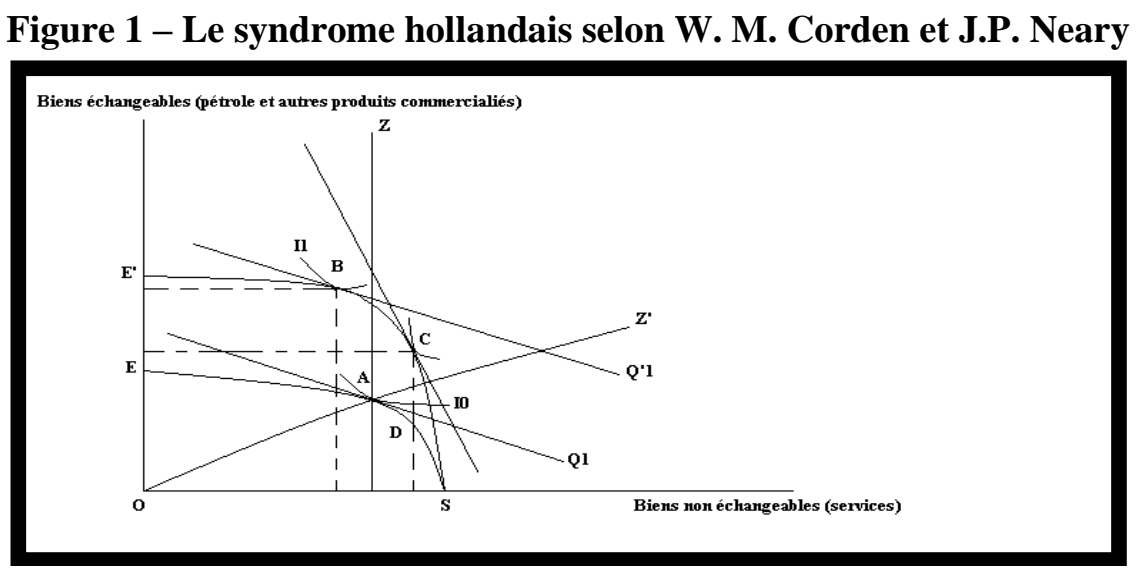

Source : W. M. Corden et J.P. Neary

Ainsi, sur la figure 1, se trouve en ordonnée le bien composite résultant de l'agrégation, en termes de l'échange constants, des productions Sc et Se. En fait, lorsque les termes de l'échange (TDE) sont fixés, le secteur où se produit le boom $(S c)$ et celui des autres biens échangeables hors boom $(\mathrm{Se})$ peuvent être agrégés dans un même secteur (celui des biens échangeables) pour former un bien composite commercialisé. L'équilibre initial se situe au point A selon un taux de change réel (prix relatif des biens exposés et des biens abrités ou non exposés) où la courbe d'isoproduit $\mathrm{I} 0$ est tangente à la courbe des possibilités de production $(E S)$.

À la suite du boom dans Sc (secteur pétrolier ici), ce dernier, qui prend la forme d'un accroissement de productivité neutre au sens de J.R. Hicks, se traduit par le passage de la courbe de transformation (courbe des possibilités de production) de ES à E'S (cf. figure 1). En supposant invariant le prix relatif des biens abrités et des biens exposés, la production se déplace de A à $\mathrm{B}$. La hausse de la rentabilité dans $\mathrm{Sc}$ est responsable d'un prélèvement du facteur mobile $(L)$ dans Se et Sn et donc, du déclin de l'offre dans ces derniers. Le secteur en boom $(S c)$ capte l'essentiel du travail, car les opportunités de salaires (et de profit) y sont plus élevées que dans les deux autres secteurs (Se et Sne). Ainsi, le nouveau point d'équilibre de production correspondant à un accroissement des biens échangeables de EE' se situe en B sur la nouvelle courbe d'isoproduit I1.

Dans ce cadre, l'accroissement des revenus tirés du boom va induire une hausse de la demande des biens abrités qui voient ainsi leurs prix augmenter. Dès lors, l'appréciation du taux de change réel $(T C R=$ $\left.R=S^{*} P E / P N E\right)$ va, via l'effet Stolper-Samuelson', provoquer la croissance de la production des biens abrités ou non échangeables (Sne) et une baisse de celle des autres biens échangeables hors boom $(\mathrm{Se})$, de sorte que le point d'équilibre se déplace, cette fois, du point $\mathrm{B}$ à celui $\mathrm{C}$. Le relèvement du taux de change réel $(R)$ permet donc à Sne de compenser une partie de son déclin initial en prélevant à son tour du facteur mobile $(L)$ dans le reste de l'économie, et notamment dans Se qui voit sa situation se détériorer davantage. 
Ce premier mécanisme, attribuable à la ponction initiale en ressources productives exercée par Sc, est nommé, par W.M. Corden et J.P. Neary, d' « effet de mouvement des ressources » et renvoie au transfert du facteur mobile $(L)$ vers le secteur en boom $(S c)$ et celui des biens non échangeables (Sne). Si le secteur en boom n'est pas une enclave, il exerce des effets sur les ressources productives et plus précisément sur la mobilité du travail. Il y a un accroissement de la demande de travail dans le secteur pétrolier (minier) et dans celui des biens non échangeables. Comme l'offre de travail est donnée (hypothèse de plein-emploi), il en résulte un manque de main-d'œuvre dans le secteur produisant les autres biens échangeables hors pétrole ( $\mathrm{Se}$ ) qui voit, par conséquent, sa production baisser. Au point $\mathrm{C}$, la production des biens échangeables hors boom $(\mathrm{Se})$ est plus faible qu'au point initial $(A)$ et celle des biens non échangeables (Sne) devient plus importante. La contraction de la production des autres biens échangeables hors boom $(\mathrm{Se})$ est illustrée par le mouvement de $\mathrm{A}$ à $\mathrm{D}$, le long de la frontière de production $(E S)$. C'est ce glissement du point d'équilibre de production (optima de production) le long de la frontière des possibilités de production $(F P P)$ que W.M. Corden et J.P. Neary qualifient de «désindustrialisation $»$.

Toutefois, s'il est présumé que le revenu du secteur en boom est dépensé pour partie au moins en biens et services abrités, un second mécanisme (l'effet de «dépense ») entre, de ce fait, en jeu. Afin de bien l'isoler du précédent, il est présumé que Sc constitue une enclave dans l'économie et ne partage donc aucun facteur productif avec les autres secteurs. Dans ce cadre, le point de production immédiat après le choc se localise à la verticale de $\mathrm{A}$. Avec l'invariance de l'offre des biens non échangeables, une demande excédentaire apparaitra dans Sne. L'appréciation du TCR qui en découle conduit à l'expansion du secteur abrité (Sne) et à la contraction de celui exposé hors boom $(\mathrm{Se})$.

Cet effet de «dépenses » a donc trait à l'utilisation des revenus issus du boom. À la suite du choc positif dans le secteur pétrolier $(S c)$, l'excédent de la balance commerciale (courante) qui en résulte va conduire à une hausse du revenu global. Dans ce cadre, si tout ou partie de ce revenu global est dépensé en biens du secteur non exposé (Sne) et si ces derniers ne sont pas des biens inférieurs, il va advenir une expansion de la demande des biens et services non exposés. Devant une situation d'excès de la demande sur l'offre, il y aura, indépendamment de toute réallocation des ressources, une hausse des prix domestiques des biens non échangeables. Partant de là, le prix relatif va baisser et traduire une appréciation du taux de change réel qui entraînera un squeeze (contraction) de la production des biens échangeables autres que ceux du secteur échangeable en boom. Et, face à cette baisse de la production des biens échangeables du secteur hors boom (agriculture, élevage, pêche), la demande intérieure stimulée par l'augmentation du revenu réel se tourne vers les importations de ces derniers et provoque une détérioration du solde extérieur en biens alimentaires.

\section{CE QUE L'ON VOUDRAIT SAVOIR SUR LA RELATION ENTRE LE BOOM DU PÉTROLE ISSU DE LA POLITIQUE PÉTROLIÈRE DE LA DÉCENNIE 1970 ET LA «MALADIE NÉERLANDAISE » DE L'ÉCONOMIE GABONAISE}

Dans ce cadre, la question spécifique de recherche est donc celle de savoir si le boom du pétrole issu de la politique pétrolière de la décennie 1970 n'a-t-il pas modifié la structure de l'économie gabonaise au détriment du hors pétrole (bois, mines, agriculture).

Sur la base de la théorie du «syndrome hollandais », il est approprié de savoir si le boom $\mathrm{du}$ pétrole issu de la politique pétrolière de la décennie pétrolière (large ouverture et fiscalité attractive) de la décennie 1970 a, au travers des effets de «mobilité des facteurs productifs » et de «dépenses », engendré une modification de la base économique du Gabon au détriment du hors pétrole (bois, mines, agriculture) à partir des années 1980. L'étude d'asymétrie temporelle entre la politique pétrolière de la décennie 1970 et le changement de la structure de l'économie gabonaise au profit du «pétrole» à partir des années 1980 s'inscrit notamment dans une approche théorique «pluraliste ». Cette dernière consiste en ce que le boom du pétrole ait, au travers des hauts revenus de travail et de capital 
versés, attiré par-devers elle les ressources productives du hors pétrole (sylviculture, mines, agriculture). Par ailleurs, en raison des fortes recettes d'exportations pétrolières en devises (dollar américain notamment) sans cesse monétisées dans l'économie, le secteur pétrolier en expansion a engendré une hausse des liquidités en circulation, une augmentation des encaisses monétaires détenues par les agents non bancaires (ménages, entreprises, gouvernement), une croissance de la dépense intérieure (consommation, investissement, dépenses publiques). La situation d'excès de cette dépense domestique sur l'offre domestique a provoqué une hausse du niveau des prix des biens et services domestiques. Partant de là, l'inflation générée a entraîné une appréciation réelle de la monnaie nationale $(T C R \downarrow=R \downarrow=$ $S * P E / P N E \uparrow)$, une perte de compétitivité-prix internationale et un déclin relatif pour les produits hors boom exposés tels que le bois, les mines, et notamment l'agriculture ${ }^{10}$.

Toutefois, si de nombreux chercheurs se sont penchés sur le lien entre le « boom du pétrole » et la «maladie néerlandaise » d'une économie, cette problématique a rarement été abordée dans le cadre spécifique du Gabon. Aussi, se pose-t-on la question de savoir si les conclusions de ces chercheurs peuvent, en la matière, s'appliquer

\section{LE CADRE DE RÉFÉRENCE}

Il s'agit ici de mettre en exergue l'impact (effet non désiré) de la politique pétrolière de la décennie 1970 en matière de modification de la structure de l'économie gabonaise.

Le cadre de référence de la recherche menée ici porte sur la relation entre le «boom du pétrole » issu de la politique pétrolière de la décennie 1970 et la « maladie néerlandaise » de l'économie gabonaise. Il repose sur la vérification d'une relation asymétrique temporelle entre le «boom du pétrole » et la «maladie néerlandaise » de l'économie gabonaise au travers des effets de «déplacement des ressources productives » et de « dépenses ». L'effet de «déplacement des ressources productives » renvoie au transfert du facteur mobile $(L)$ vers le secteur en boom $(S c)$ et celui des biens non échangeables $(S n e)$. L'effet de « dépenses » a trait à l'utilisation des énormes revenus issus du boom dans le cas gabonais? Autrement dit, n'est-il pas justifié, au regard d'un manque de travaux scientifiques en la matière sur le Gabon, de mener de nouvelles recherches?

Dans ce cadre, la question spécifique de recherche est donc celle de savoir si le boom du pétrole issu de la politique pétrolière de la décennie 1970 n'a-t-il pas modifié la structure de l'économie gabonaise au détriment du hors pétrole (bois, mines, agriculture) à partir des années 1980 ?

C'est donc ici une problématique de recherche qui est d'une grande pertinence ${ }^{11}$ quant à sa contribution à la fois théorique, méthodologique et économétrique. Son apport théorique concerne la contribution de la théorie du «syndrome hollandais » à la compréhension de la métamorphose de la base économique du Gabon dès les années 1980. Son apport méthodologique repose sur l'opérationnalisation des concepts clés (effet de déplacement des ressources, effet de dépenses) en vue d'une meilleure observation sur le terrain des phénomènes en relation avec les dimensions et indicateurs qui y sont afférents. Son apport économétrique est de mettre en exergue, au moyen d'études sérielles, l'impact des effets de « mobilité des ressources » et de « dépenses » du boom pétrolier sur les autres secteurs d'activité.

pétrolier dans les secteurs des biens non exposés (logement, santé) avec son corollaire d'expansion de la demande intérieure et des prix domestiques de ces derniers. Ces deux concepts permettent alors de démontrer le caractère exclusif des exportations de l'or noir sur les autres produits d'exportations de l'économie gabonaise. Ils désignent l'ensemble des effets préjudiciables créés dans une économie par l'expansion d'un secteur et se traduisent par de brusques modifications dans l'attribution des ressources, des changements de la structure sectorielle du système productif, les mouvements des prix relatifs étant au centre de ces distorsions sectorielles.

Cette étude s'appuyant sur les résultats de travaux antérieurement menés sous d'autres cieux, l'existence d'une asymétrie temporelle entre les deux phénomènes (boom du pétrole et maladie néerlan- 
daise de l'économie gabonaise) est présumée, et ce, à l'aulne de la théorie du « syndrome hollandais ». Il s'agit ici de mettre en exergue l'impact (effet non désiré) de la politique pétrolière de la décennie 1970

\section{LA MÉTHODOLOGIE}

\section{L'hypothèse de la « maladie néerlandaise » effective de l'économie gabonaise positivement corrélée au «boom du pétrole » issu de la politique pétrolière.}

La méthodologie utilisée en vue d'atteindre l'objectif de recherche (validité interne) porte essentiellement sur la spécification de l'hypothèse de recherche, la détermination des indicateurs, du devis de recherche et la discrimination des stratégies de collecte de données appropriées.

Ainsi, la réponse à la question de recherche conduit à s'inscrire sous l'hypothèse de la «maladie néerlandaise » effective de l'économie gabonaise positivement corrélée au «boom du pétrole » issu de la politique pétrolière menée par les autorités

\section{RÉSULTATS ET DISCUSSION}

5.1. La base pétrolière de l'économie gabonaise après le choc pétrolier de la décennie 1970

5.1.1. La structure économique du Gabon avant le choc pétrolier de la décennie 1970: une hypertrophie du hors pétrole et atrophie du pétrole en matière de modification de la structure de l'économie gabonaise au profit du pétrole et au détriment des autres produits hors boom exposés à la concurrence internationale.

publiques dans la décennie 1970. Pour y arriver, les principaux indicateurs utilisés pour mettre en exergue les différents effets de «déplacement des ressources productives », de «dépenses » sont le produit intérieur brut $(P I B)$, la valeur ajoutée pétrolière $(V A P)$, la valeur ajoutée agricole $(V A A)$, les recettes d'exportations pétrolières (REXPET), la production de cacao (PRODCACAO), les frais de personnel $(F P)$, la main-d'œuvre agricole $(M O A)$, l'indice des prix à la consommation (IPC), le taux de change effectif réel (TCER). Le devis de la présente recherche scientifique se veut hypothéticodéductif et explicatif. Il vise simplement à établir une relation d'asymétrie temporelle entre le boom du pétrole et la maladie néerlandaise de l'économie gabonaise. La collecte de données s'est appuyée sur deux instruments, dont l'analyse des documents et celle des statistiques.

$\mathrm{Au}$ Gabon, le hors pétrole qui regroupe l'ensemble des activités économiques (bois, mines, agriculture) en dehors du pétrole représentait $79,7 \%$ des exportations contre 20,3\% pour le pétrole en 1960.

Graphique 1 - Exportations du hors pétrole et pétrole (en \%), 1960

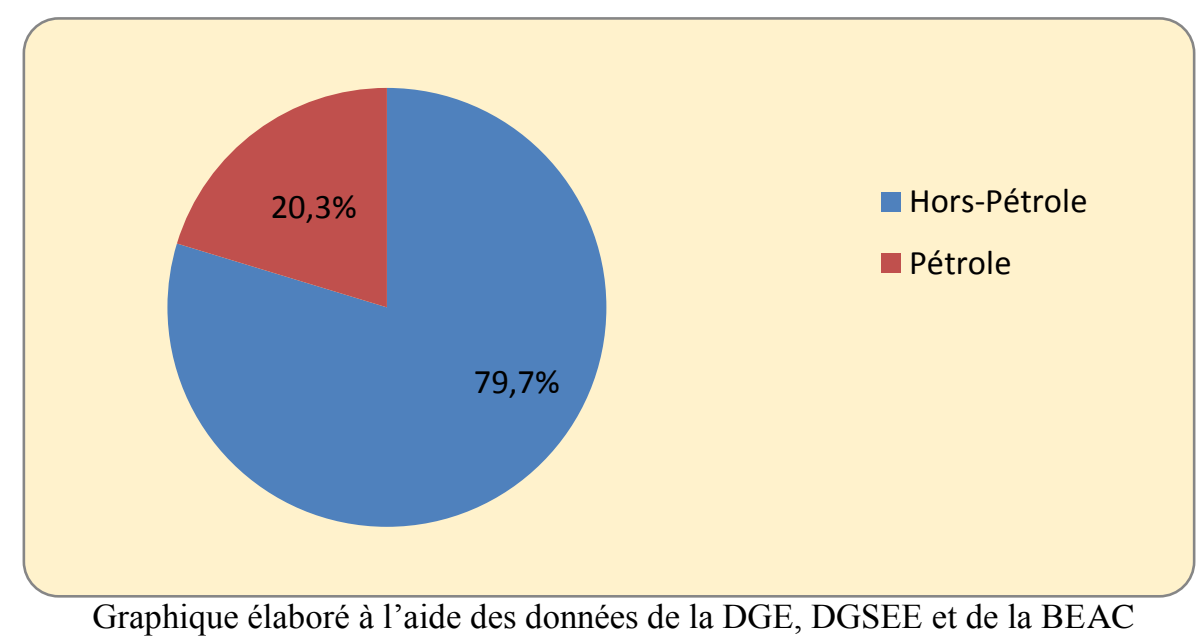


Dans ce cadre, le bois $^{12}$, avec son potentiel exploitable de 300 millions de mètres cubes et sa couverture des trois quarts du pays, était, de 1920 à
1970, le produit dominant de l'économie gabonaise et représentait $69,9 \%$ du total des exportations contre $20,3 \%$ pour le pétrole en 1960 .

Graphique 2 - Exportations du bois et du pétrole (en \%), 1960

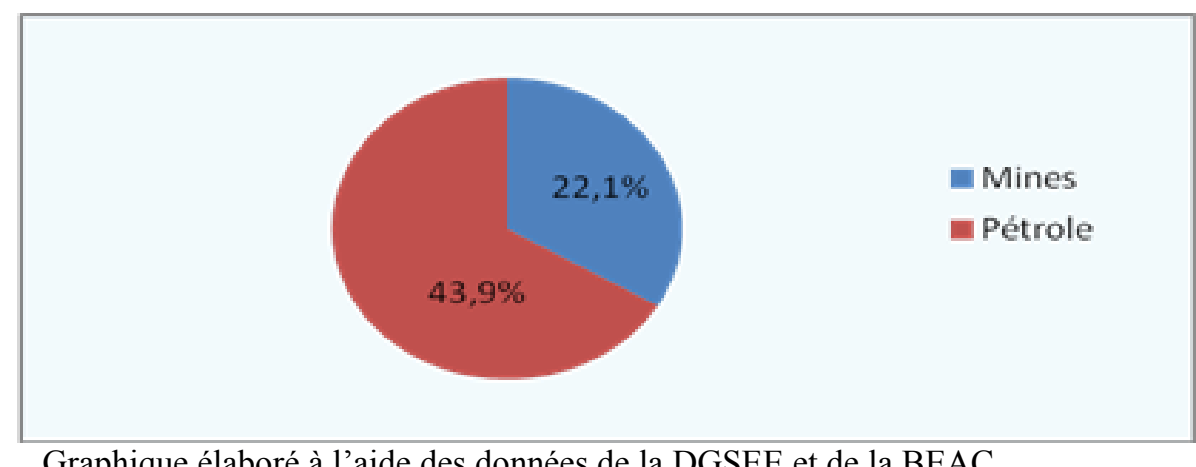

Graphique élaboré à l'aide des données de la DGSEE et de la BEAC

Les mines qui regroupent l'uranium et le manganèse correspondaient, en 1971, à 22,1\% des exportations du Gabon.

Graphique 3 - Exports des mines et du pétrole (en \%), 1971

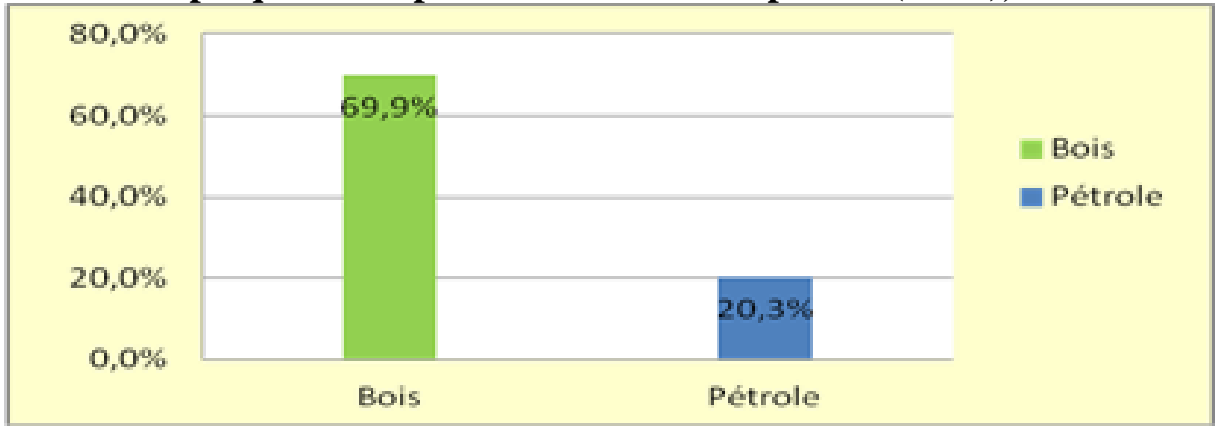

Graphique élaboré à l'aide des données de la DGSEE et de la BEAC

L'agriculture qui était surtout le fait de petites exploitations familiales, représentait, en 1960, $9,8 \%$ du total des exportations gabonaises pour des raisons notamment de carence de moyens de production modernes, de manque d'organisation, d'exode rural, de dysfonctionnement de la caisse de stabilisation.

Graphique 4 - Exports des produits agricoles et du pétrole (en \%), 1960

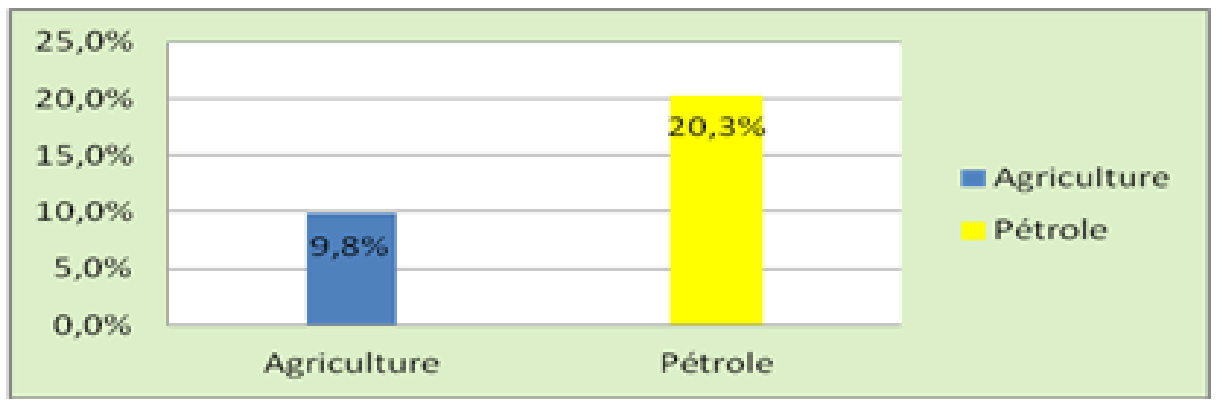

Graphique élaboré à l'aide des données de la DGSEE et de la BEAC 
5.1.2. La structure de l'économie gabonaise après le choc de la décennie 1970 : une atrophie du hors pétrole et hypertrophie du pétrole

En raison de la politique pétrolière menée par les autorités publiques ayant, dans la décennie 1970, consisté en une large ouverture du champ pétrolier aux multinationales pétrolières et en une offre de conditions fiscales attractives, la base de l'économie gabonaise avait fini par se modifier au profit du «pétrole » et au détriment du « hors pétrole ». Avec les énormes recettes pétrolières générées par la hausse $\mathrm{du}$ cours international du pétrole (2,08 USD/baril en 1971; 12,59 USD en 1977; 34,21 USD/baril en 1982; soit une hausse globale de $+1544,7 \%$ sur la période 1971-82), le gouvernement gabonais avait libéralisé un peu plus le secteur pétrolier et adouci la fiscalité pétrolière en vue de doper la production de l'or noir gabonais (5,7 millions de tonnes en 1971; 11,2 millions de tonnes en 1977; 13,5 millions de tonnes en 1990; 18,2 millions de tonnes en 1995; soit une hausse globale de 219,3\% sur la période 1971-95). Malheureusement, cette politique pétrolière eut pour impact de faire naître et renforcer la «maladie néerlandaise » de l'économie par le leadership du produit «pétrole» dans les agrégats économiques. De la sorte, le secteur hors pétrole avait souffert du boom pétrolier de la décennie 1970. L'appréciation du taux de change effectif réel (83,4 en 1970; 81,6 en 1974; 74,9 en 1984; 67,7 en 1990) ${ }^{13}$ du franc CFA induite par la hausse du cours et de la production de l'or noir avait brisé sa compétitivité-prix internationale.

\section{Graphique 5 - Évolution du TCER du F. CFA du Gabon de 1970 à 1990}

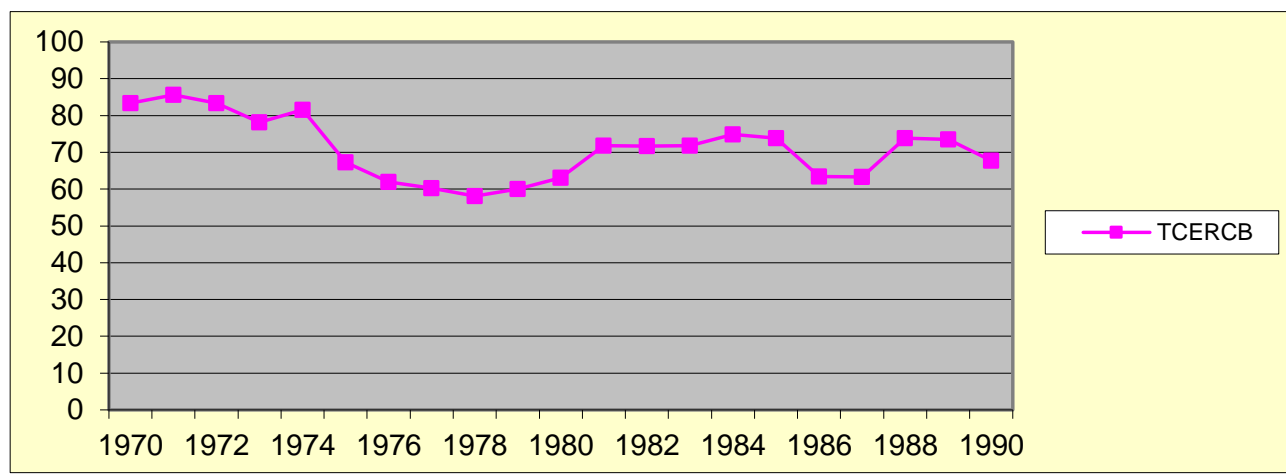

Source : Okoué Edou, J.J.R., 2007, p. 58

* Une baisse du TCER = appréciation réelle du franc CFA

* Une hausse du TCER = dépréciation réelle du franc CFA

Il s'en était suivi une atrophie de ce dernier dans les exportations totales $(17,6 \%$ en 1990 contre $79,7 \%$ en 1960) pendant que le secteur pétrolier s'hypertrophiait (82,4\% en 1990 contre $20,3 \%$ en 1960).

\section{Graphique 6 - Exports du hors pétrole et du pétrole, 1960 et 1990}

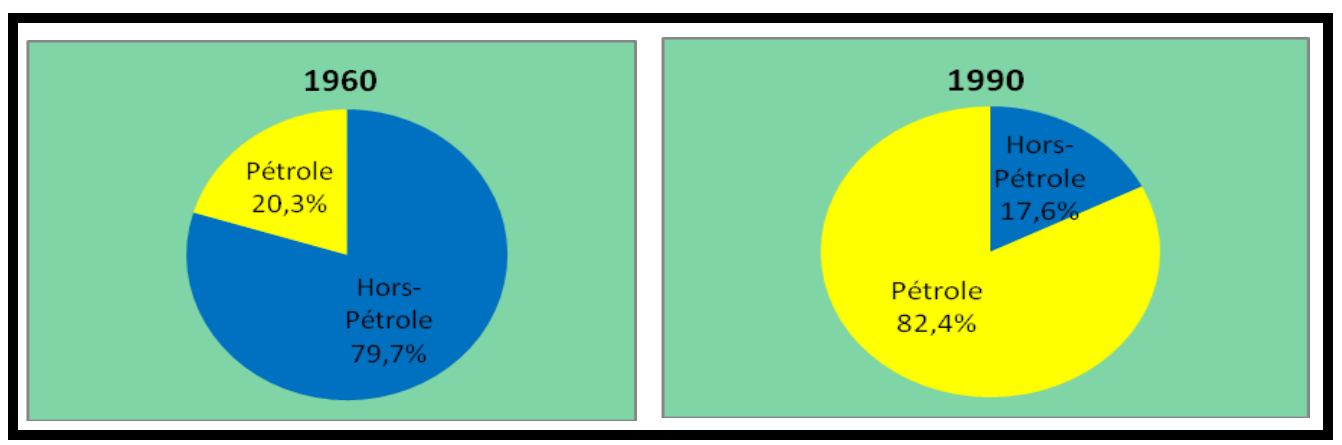

Graphique élaboré à l'aide des données de la DGE, DGSEE et de la BEAC 
Avec le boom pétrolier de 1973-74, la contribution de la sylviculture dans les exportations totales n'a cessé de baisser durant la période de croissance des recettes pétrolières.

\section{Graphique 7 - Exportations du bois et du pétrole, 1960 et 1990}

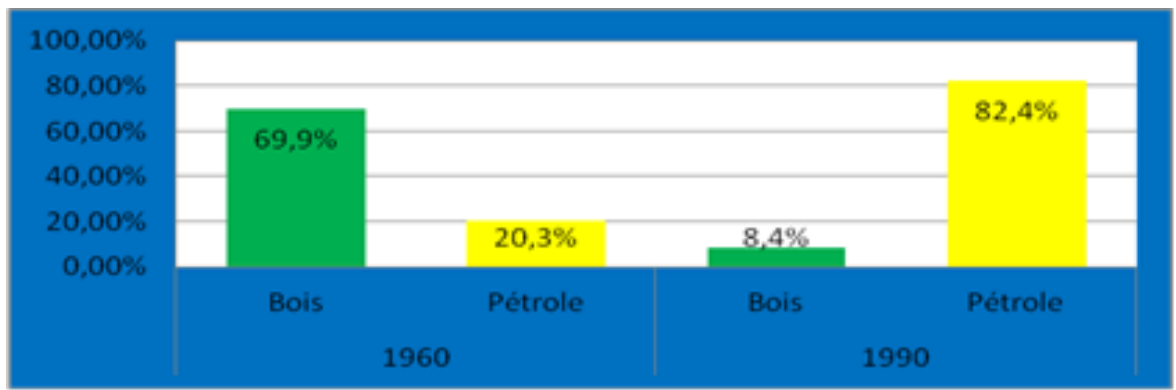

Graphique élaboré à l'aide des données de la DGSEE et de la BEAC

À cet égard, elle a vu sa part drastiquement chuter de $69,9 \%$ en 1960 à 30,2\% en 1971 puis à $8,4 \%$ en 1990 et, a ainsi dû céder sa place de produit leader de l'économie gabonaise au pétrole. Ainsi, l'appréciation du TCER du franc CFA $(67,7$ en 1990 contre 83,4 en 1970) induite par le boom pétrolier avait provoqué une hausse des coûts de l'or vert gabonais et donc, une chute drastique de ses exportations.

Si la branche d'activité « mines » contribuait pour $22,1 \%$ dans les exportations du Gabon en 1971, son poids au sein de cet agrégat économique a connu, avec le boom pétrolier de 1973-74, une baisse substantielle qui l'a stabilisée à 8,4\% en 1990.

\section{Graphique 8 - Exports des mines et du pétrole (en \%), 1971 et 1990}

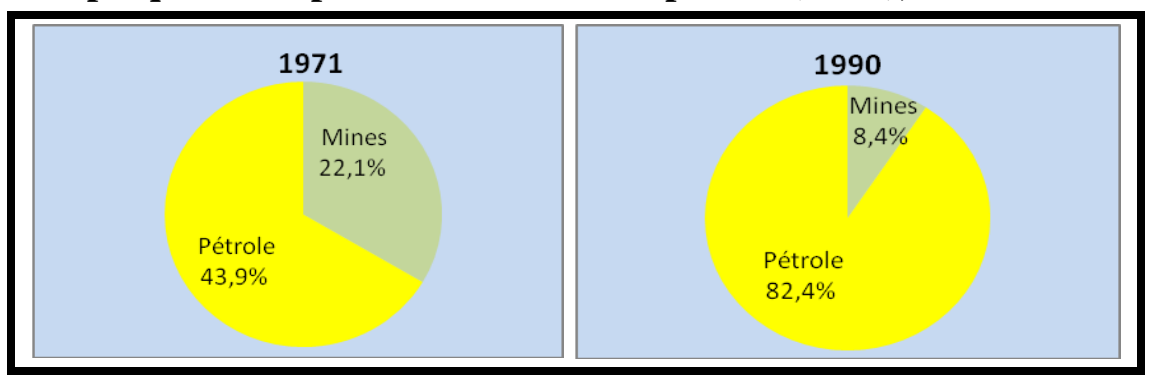

Graphique élaboré à l'aide des données de la DGSEE et de la BEAC

Les revenus pétroliers ont suscité, avec le boom de l'or noir, des rentrées massives de devises, une hausse du niveau moyen des prix et une appréciation réelle du franc CFA. Partant de là, l'agriculture, dont le poids au sein de l'économie gabonaise était déjà faible, a dû pâtir davantage des effets pernicieux de la baisse de compétitivité générale et de la modification des prix relatifs.

\section{Graphique 9 - Exports des produits agricoles et du pétrole (en \%), 1960 et 1990}

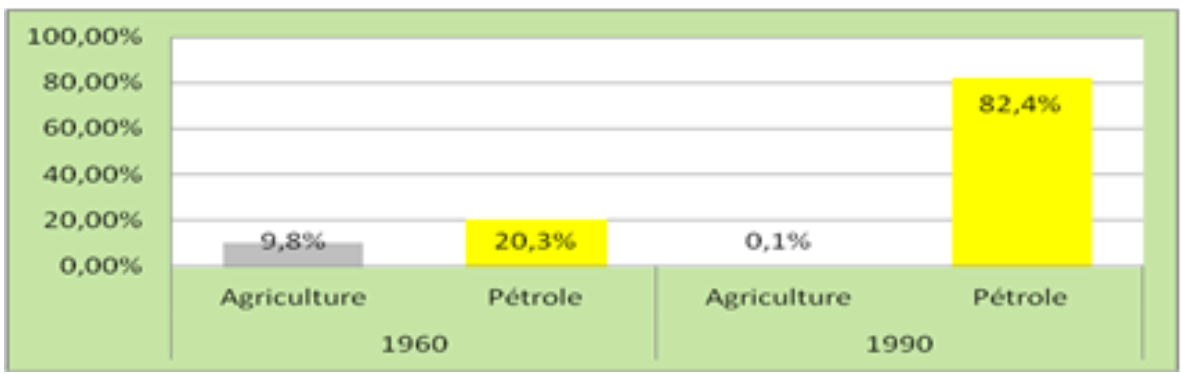

Graphique élaboré à l'aide des données de la DGSEE et de la BEAC 
En effet, avec la modification à la baisse des prix relatifs, la contribution du secteur agricole dans le total des exportations est passée de $9,8 \%$ en 1960 à $0,10 \%$ en 1990 . Malgré le leitmotiv du gouvernement de faire de l'agriculture, la «priorité des priorités » ou la «base du développement », elle a toujours été reléguée à la lisière de l'autosubsistance. Cependant, l'impact du boom pétrolier a été différent dans chacune des quatre sous-branches (agriculture de subsistance, agriculture vivrière d'import-substitut, agriculture d'export et agroindustrie parapublique) ${ }^{14}$.

De la sorte, avec l'expansion de sa production, la part relative du pétrole aux exports totaux a atteint des proportions telles que ce produit est devenu celui leader dans les principaux agrégats économiques (PIB, recettes d'exportations, recettes budgétaires) du Gabon.

Tableau 1 - Parts du pétrole dans les agrégats économiques, 1980-2013

\begin{tabular}{|c|c|c|c|c|c|c|c|c|}
\hline Années & 1980 & 1990 & 2000 & 2009 & 2010 & 2011 & 2012 & 2013 \\
\hline PIB (Mds F.CFA) & 969 & 1477 & 3577 & 5702 & 7201 & 8867 & 9527 & 10327 \\
\hline VAPET $(M d s F . C F A)$ & 392 & 485 & 1736 & 3475 & 4238 & 4936 & 5553 & 6200 \\
\hline VAPET/PIB (\%) & 40,4 & 32,8 & 48,5 & 60,9 & 58,8 & 55,7 & 58,3 & 60 \\
\hline REEXP (Millions USD) & 2633 & 2619 & 3219 & 5922 & 7464 & 10463 & 9927 & 10501 \\
\hline REEXPPET (Millions USD) & 1930 & 1885 & 2574 & 4909 & 6512 & 9382 & 8760 & 8879 \\
\hline REEXPPET/REEXP (\%) & 73,3 & 72,0 & 79,9 & 82,3 & 87,2 & 89,7 & 88,2 & 84,5 \\
\hline REBUD $(M d s F . C F A)$ & - & - & 1207 & 1685 & 1834 & 2469 & 2546 & 2547 \\
\hline REBUDPET (Mds F.CFA) & - & - & 815 & $\mathbf{8 3 7}$ & 986 & 1372 & 1460 & 1408 \\
\hline REBUDPET/REBUD (\%) & - & - & 67,5 & 49,7 & 53,8 & 55,6 & 57,3 & 55,3 \\
\hline
\end{tabular}

Tableau élaboré à l'aide des données du FMI (Rapport du FMI no 13/55, pp. 30-38)

$\mathrm{PIB}=$ Produit intérieur brut; VAPET $=$ Valeur ajoutée pétrolière; $\mathrm{REEXP}=$ Recettes d'exportations totales REEXPPET $=$ Recettes d'exportations pétrolières; REBUD $=$ Recettes budgétaires totales;

REBUDPET - Recettes budgétaires pétrolières.

Ainsi, le produit «pétrole » est en position de tête dans les recettes d'exportations et représente en moyenne $86,4 \%$ du total sur la période 2009-2013 contre $15,6 \%$ pour le «hors pétrole ». Il est le produit dominant dans les recettes budgétaires et représente en moyenne $54,3 \%$ du total sur la période $2009-2013$ contre $45,7 \%$ pour le «hors pétrole ». Le secteur pétrolier est aussi la première source de création de richesse et représente en moyenne 58,7 \% du PIB sur la période 2009-2013 contre 41,3\% pour le «hors pétrole ». En 2013 spécifiquement, le pétrole, principale ressource du Gabon (4e producteur d'Afrique subsaharienne) avec un bassin sédimentaire de $250000 \mathrm{~km}^{2}$ et une production d'environ 289700 barils/jour, contribuait à $60 \%$ au PIB, intervenait à plus de $80 \%$ dans les recettes d'exportations et générait plus de $55 \%$ des recettes budgétaires.

\subsection{La justification économétrique des effets de} « déplacement des ressources » et de « dépenses »

5.2.1. La justification économétrique de l'effet de « déplacement des ressources »

Selon 1'effet de «déplacement des ressources productives », la hausse de la rentabilité dans le secteur exposé en boom est responsable d'un prélèvement $\mathrm{du}$ facteur mobile des secteurs exposés hors boom (mines, agriculture, forêt). Ce secteur exportateur en expansion capte l'essentiel du travail, car les opportunités de salaires y sont plus élevées que dans les autres. Comme le pleinemploi du travail est présumé, il en résulte un manque de main-d'œuvre dans les secteurs d'activité exposés hors boom et donc, une baisse de leur production qualifiée de «désindustrialisation $»^{15}$ ou de $«$ désagriculturisation $»^{16}$.

Dans ce cadre, l'étude économétrique de cet effet de «réallocation des ressources productives» a conduit au choix de la branche pétrolière (branche exposée à la concurrence internationale en boom) et de celle agricole (branche exposée au commerce international hors boom). Plus précisément, les variables arrêtées sont la valeur ajoutée pétrolière $(V A P)$ et la main-d'œuvre agricole $(M O A)$.

Afin de faire ressortir la corrélation négative entre la main-d'œuvre agricole $(M O A)$ et la valeur ajoutée pétrolière $(V A P)$, ce modèle économétrique a été construit ${ }^{17}$ (voir les séries chronologiques afférentes aux variables MOA et VAP en annexe). 
$\mathrm{MOA}_{\mathrm{t}}=\mathrm{aVAP}_{\mathrm{t}}+\mathrm{C}+\mathrm{Ut}$ tels que MOA $=$ main-d'œuvre agricole $=$ explanandum; VAP $=$ valeur ajoutée pétrolière $=$ explanans; $\mathrm{a}=$ paramètre de comportement de MOA consécutive à la variation de VAP; $\mathrm{C}=$ constante non nulle; $\mathrm{U}=$ résidu; $\alpha=$ risque d'erreur $=5 \% ; \mathrm{T}=$ nombre d'observations $=15$ et $\mathrm{k}=$ nombre de variables explicatives (terme constant y compris) $=2$.

Il ressort, avec un seuil de confiance $((=1-\alpha)$ de $95 \%$, ce modèle économétrique estimé.

$$
\begin{gathered}
\mathrm{MOA}_{\mathrm{t}}=-0.171 \mathrm{VAP}_{\mathrm{t}}+2323.76+\mathrm{e}_{\mathrm{t}} \\
\left(-4.583^{*}\right) \quad(74.155)
\end{gathered}
$$

Ainsi, il existe bien une corrélation négative entre MOA et VAP $(\hat{a}=-0.171)$. L'expansion de la valeur ajoutée pétrolière a bien agi négativement sur la branche agricole en y captant l'essentiel de sa main-d'œuvre attirée par les opportunités de salaires élevés. La statistique $F$ de Fisher indique que le modèle est globalement significatif. Pour $\mathrm{k}=$ $2, \mathrm{~T}=15$ et $\alpha=0.05$, la probabilité critique (probabilité d'acceptation de l'hypothèse HO) de ce test est inférieure à $5 \%$ (prob (F-statistic) = $0.000513<5 \%$ ), et ce, même si le coefficient de détermination $\left(R^{2}\right)$ corrobore faiblement ce bon résultat de l'ajustement global du modèle (son pouvoir explicatif est de 61,7\%). Le $t$ de Student (y compris celui du terme constant, C), de la variable VAP a une probabilité critique inférieure à $5 \%$ $($ prob $(t$-Statistic $)=0.0005<5 \%)$. Autrement dit, le coefficient de régression de MOA sur VAP est significativement différent de 0 . Le boom du pétrole a bien contribué au déclin de la branche exposée hors boom agricole $(\hat{a}=-0.171)$ par la captation de sa main-d'œuvre. Le coefficient de corrélation linéaire $\left(R_{M O A, V A P}=-0.785\right)$ corrobore assez fortement cette relation réciproque négative entre MOA et VAP. La statistique $d$ de Durbin-Watson $(d=2.13)$ est bonne (elle ne semble pas présager d'autocorrélation des résidus). Pour m (nombre de variables explicatives, terme constant exclu) $=1, \mathrm{~T}$ (nombre d'observations) $=15$ et $\alpha=0.05=5 \%$, il advient des $\mathrm{d}_{1}=1.08$ et $\mathrm{d}_{2}=1.36$ avec $\mathrm{d}_{2}=1.36<\mathrm{d}$ $=2.13<4-\mathrm{d}_{2}=2.64$.

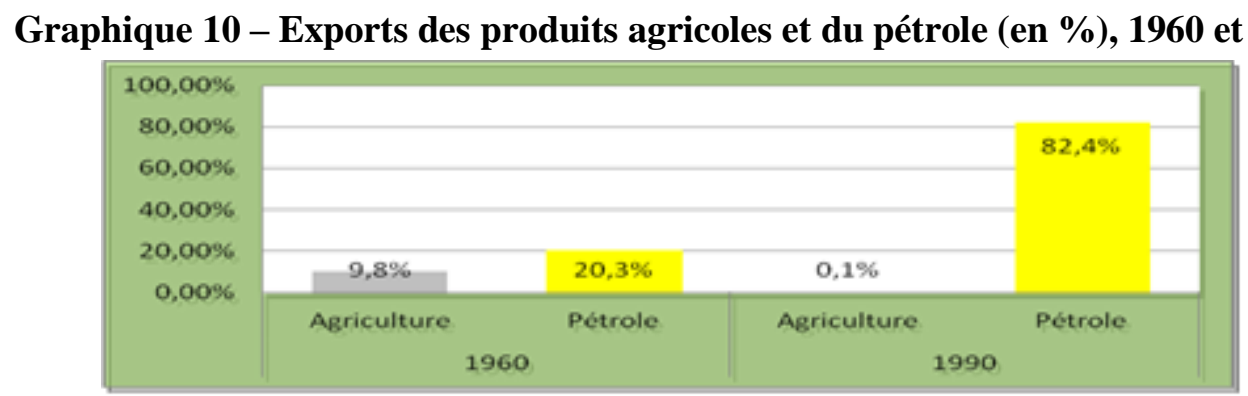

Graphique élaboré à l'aide des données de la DGE, DGSEE et de la BEAC

Somme toute, la branche pétrolière a capté l'essentiel du travail de la branche agricole par son expansion et les hauts salaires ${ }^{18}$ versés pour attirer cette ressource productive vers elle.
Ainsi, soit l'évolution des frais de personnel des différentes branches d'activité sur la période 19881991.

Tableau 2 - Frais du personnel dans les principales branches d'activités, 1988-1991

\begin{tabular}{|l|r|r|r|r|}
\hline Branches\Années & 1988 & 1989 & 1990 & 1991 \\
\hline 1. Pétrole & 51.303 & 68.295 & 65.940 & 68.900 \\
\hline 2. Forêts et industries du bois & 18.832 & 18.920 & 18.591 & 18.119 \\
\hline 3. Mines et carrières & 22.446 & 19.071 & 18.972 & 21.956 \\
\hline 4. Agriculture, chasse et pêche & 9.612 & 9.907 & 9.775 & 10.634 \\
\hline 5. Industries agroalimentaires & 13.261 & 12.613 & 13.483 & 15.077 \\
\hline 6. Autres industries & 16.814 & 17.689 & 18.592 & 19.964 \\
\hline 7. Énergie (électricité et eau) & 20.035 & 19.545 & 20.439 & 21.160 \\
\hline 8. BTP & 25.771 & 22.468 & 21.867 & 23.085 \\
\hline 9. Transports et télécommunications & 54.716 & 56.054 & 59.789 & 64.941 \\
\hline 10. Commerce & 32.582 & 30.951 & 32.306 & 32.455 \\
\hline 11. Institutions financières & 16.698 & 17.149 & 17.579 & 17.579 \\
\hline 12. Autres services & 44.977 & 43.233 & 41.808 & 42.289 \\
\hline 13. Administration & 161.698 & 160.449 & 177.850 & 187.666 \\
\hline
\end{tabular}

Tableau élaboré à l'aide des données des TBE unités : Millions francs CFA 
Soit l'évolution des effectifs employés desdites différentes branches d'activité sur la même période 1988-1991.

Tableau 3 - Effectifs employés dans les principales branches d'activités, 1988-1991

\begin{tabular}{|c|c|c|c|c|}
\hline Branches\Années & 1988 & 1989 & 1990 & 1991 \\
\hline 1. Pétrole & 2.645 & 2.797 & 2.903 & 2.672 \\
\hline 2. Forêts et industries du bois & 6.341 & 5.841 & 5.794 & 4.829 \\
\hline 3. Mines et carrières & 2.747 & 2.588 & 2.580 & 2.392 \\
\hline 4. Agriculture, chasse et pêche & 2.279 & 2.181 & 2.260 & 2.290 \\
\hline 5. Industries agroalimentaires & 4.889 & 4.619 & 4.464 & 3.775 \\
\hline 6. Autres industries & 3.014 & 2.929 & 2.985 & 2.727 \\
\hline 7. Énergie (électricité et eau) & 2.075 & 1.991 & 1.954 & 1.792 \\
\hline 8. BTP & 4.606 & 3.839 & 3.830 & 4.422 \\
\hline 9. Transports et télécommunications & 9.789 & 9.576 & 9.542 & 9.405 \\
\hline 10. Commerce & 2.342 & 2.292 & 2.297 & 2.074 \\
\hline 11. Institutions financières & 8.531 & 7.924 & 7.483 & 7.457 \\
\hline 12. Autres services & 6.203 & 6.028 & 5.607 & 5.242 \\
\hline 13. Administration & 41.125 & 42.252 & 42.018 & 43.108 \\
\hline
\end{tabular}

Tableau élaboré à l'aide des données TBE (en unités indiquées)

Du ratio frais de personnel sur effectifs employés pour obtenir le coût salarial unitaire, il ressort que la branche pétrolière en boom était celle qui versait les rémunérations les plus élevées.

Tableau 4 - Coût salarial unitaire (FP/effectifs employés) des branches d'activités, 1988-1991

\begin{tabular}{|c|c|c|c|c|}
\hline Branches\Années & 1988 & 1989 & 1990 & 1991 \\
\hline 1. Pétrole & 19,40 & 24,42 & 22,71 & 25,78 \\
\hline 2. Forêts et industries du bois & 2,97 & 3,24 & 3,21 & 3,75 \\
\hline 3. Mines et carrières & 8,17 & 7,37 & 7,35 & 9,18 \\
\hline 4. Agriculture, chasse et pêche & 4,22 & 4,54 & 4,32 & 4,64 \\
\hline 5. Industries agroalimentaires & 2,71 & 2,73 & 3,02 & 3,99 \\
\hline 6. Autres industries & 5,58 & 6,04 & 6,23 & 7,32 \\
\hline 7. Énergie (électricité et eau) & 9,65 & 9,82 & 10,46 & $\mathbf{1 1 , 8 1}$ \\
\hline 8. BTP & 5,59 & 5,85 & 5,71 & 5,22 \\
\hline 9. Transports et télécommunications & $\mathbf{5 , 5 9}$ & 5,85 & 6,26 & 6,90 \\
\hline 10. Commerce & 13,91 & $\mathbf{1 3 , 5 0}$ & 14,10 & 15,65 \\
\hline 11. Institutions financières & 1,96 & 2,16 & 2,34 & 2,36 \\
\hline 12. Autres services & 7,25 & 7,17 & 7,45 & 8,10 \\
\hline 13. Administration & 3,93 & 3,79 & 4,23 & $\mathbf{4 , 3 5}$ \\
\hline
\end{tabular}

Tableau élaboré à l'aide des données des TBE (unités : Millions francs CFA)

Ainsi, en versant des rémunérations plus élevées que les autres branches de l'économie, la branche pétrolière en expansion a attiré par-devers elle le facteur travail des autres et s'est développée au détriment d'elles, et notamment de la branche agricole. Et, comme l'offre de travail était donnée ou fixe (hypothèse de plein-emploi), il a résulté un manque de main-d'œuvre dans la branche agricole qui a vu sa production baisser.

\subsubsection{La justification économétrique de l'effet de «dépenses »}

Selon W.M. Corden et J.P. Neary, si le revenu du secteur en boom est dépensé en partie au moins en biens et services abrités, un second mécanisme entre, de ce fait, en jeu. Il s'agit de l'effet de
« dépenses » lié à l'utilisation des revenus issus du secteur en expansion.

Dans le cas du Gabon, à la suite du boom dans le secteur pétrolier, l'excédent durable de sa balance commerciale avait, via la monétisation systématique des énormes recettes d'exportations pétrolières en devises, engendré la hausse des liquidités en circulation dans l'économie (masse monétaire) et du revenu global, conduit à une explosion de la demande intérieure pour les biens et services non exposés, et induit à la hausse l'indice des prix à la consommation (inflation).

L'expansion des recettes d'exportations pétrolières avait préalablement alimenté le budget de l'État et expliqué la hausse des dépenses publiques. Il faut 
souligner qu'au sortir de la colonisation, l'État était le principal investisseur et pourvoyeur de l'emploi. Malgré le dualisme de l'économie gabonaise, le pont entre le secteur « pétrole » et le secteur « hors pétrole» demeure le budget de l'État qui a été régulièrement alimenté par les recettes d'exportations pétrolières. Or, la distribution des salaires et autres avantages aux agents de l'État, l'investissement massif dans les infrastructures publiques, le subventionnement de grandes structures productrices ont fortement dopé la demande intérieure et alimenté l'inflation.

\section{Ainsi, l'expansion des recettes d'exportations du secteur « pétrole » en expansion a bien alimenté le budget de l'État et provoqué, à travers la hausse des dépenses publiques, une hausse de l'indice des prix à la consommation (IPC).}

Afin de mettre en évidence cette corrélation positive entre les dépenses publiques et les recettes d'exportations pétrolières, ce modèle économétrique a été construit (voir les séries chronologiques afférentes aux variables DEPPUB et REXPET en annexe).

DEPPUB $_{t}=$ aREXPET $_{t}+\mathrm{C}+$ Ut tels que DEPPUB $=$ dépenses publiques $=$ variable expliquée; REXPET $=$ recettes d'exportations pétrolières $=$ variable explicative; $\mathrm{a}=$ paramètre exprimant la variation de DEPPUB suite à celle de REXPET; $\mathrm{C}=$ constante non nulle; $\mathrm{U}=$ résidu; $\alpha=$ risque d'erreur $=1 \% ; \mathrm{T}=$ nombre d'observations $=$ 16 et $\mathrm{k}=$ nombre de variables explicatives (terme constant y compris) $=2$.

Il ressort, avec un seuil de confiance $((=1-\alpha)$ de $99 \%$, ce modèle économétrique estimé.

$$
\begin{aligned}
\text { DEPPUB }_{\mathrm{t}}= & 0.420 \mathrm{REXPET}_{\mathrm{t}}+272.58+\mathrm{e}_{\mathrm{t}} \\
& \left(5.475^{*}\right)
\end{aligned}
$$

Ainsi, il existe bien une corrélation positive entre DEPPUB et REXPET (le coefficient de régression a bien le signe attendu, $\hat{a}=+0.420$ ) sur la période 1982-1997. La hausse des recettes d'exportations pétrolières liée au boom de l'or noir a bien expliqué celle des dépenses publiques. La statistique $F$ de Fisher indique que le modèle est globalement bon. Pour $\mathrm{k}=2, \mathrm{~T}=16$ et $\alpha=0.01$, la probabilité critique (probabilité d'acceptation de l'hypothèse HO) de ce test est inférieure à $1 \%($ prob $(F$-statistic $)=$
$0.000082<1 \%$ ), et ce, même si le coefficient de détermination $\left(R^{2}\right)$ corrobore faiblement ce bon résultat de l'ajustement global du modèle (son pouvoir explicatif est de 68,1\%). Le $t$ de Student (y compris celui du terme constant, C), de la variable REXPET a une probabilité critique inférieure à $1 \%$ $($ prob $(t$-Statistic $)=0.0001<1 \%)$. Autrement dit, le coefficient de régression de DEPPUB sur REXPET est significativement différent de 0 . L'expansion des recettes d'exportations pétrolières liée au boom de l'or noir a bien contribué à celle des dépenses de l'État gabonais $(\hat{a}=+0.420)$. Le coefficient de corrélation linéaire $\left(R_{D E P P U B, R E X P E T}=\right.$ +0.825 ) corrobore fortement cette relation réciproque positive entre DEPPUB et REXPET. La statistique $d$ de Durbin-Watson $(d=1.156)$ est bonne (elle ne présage pas, à $\alpha=0.01=1 \%$, d'autocorrélation des résidus). Pour $\mathrm{m}=1, \mathrm{~T}=16$ et $\alpha=0.01=1 \%$, il advient des $\mathrm{d}_{1}=0.84$ et $\mathrm{d}_{2}=1.09$ avec $\mathrm{d}_{2}=1.09<\mathrm{d}=1.156<4-\mathrm{d}_{2}=2.91$.

Ainsi, l'expansion des recettes d'exportations du secteur «pétrole » en expansion a bien alimenté le budget de l'État et provoqué, à travers la hausse des dépenses publiques, une hausse de l'indice des prix à la consommation $(I P C)$ par l'excès de la demande agrégée sur l'offre agrégée.

Afin de faire ressortir la corrélation positive entre l'inflation interne et les dépenses publiques, ce modèle économétrique a été construit.

$\mathrm{IPC}_{91 \mathrm{t}}=\mathrm{aDEPPUB}_{\mathrm{t}}+\mathrm{C}+\mathrm{Ut}$ tels que $\mathrm{IPC}_{91}$ $=$ indice des prix à la consommation (base $100=$ 1991) $=$ variable expliquée; DEPPUB $=$ dépenses publiques $=$ variable explicative; $\mathrm{a}=$ paramètre exprimant le comportement de $\mathrm{IPC}_{91}$ suite à une variation de DEPPUB; $\mathrm{C}=$ constante non nulle; $\mathrm{U}$ $=$ résidu; $\alpha=$ risque d'erreur $=5 \% ; \mathrm{T}=$ nombre d'observations $=16$ et $\mathrm{k}=$ nombre de variables explicatives (terme constant y compris) $=2$.

Il ressort, avec un seuil de confiance $((=1-\alpha)$ de $95 \%$, ce modèle économétrique estimé.

$$
\mathrm{IPC}_{91 \mathrm{t}}=0.110 \mathrm{DEPPUB}_{\mathrm{t}}+17.70+\mathrm{e}_{\mathrm{t}}
$$

Ainsi, il existe bien une corrélation positive entre DEPPUB et $\mathrm{IPC}_{91}$ (le coefficient de régression a bien le signe attendu, $\hat{a}=+0.110$ ) sur la période 1970-1985. La hausse des dépenses publiques liée 
au boom du secteur pétrolier a bien alimenté une inflation interne. La statistique $F$ de Fisher indique que le modèle est globalement significatif. Pour $\mathrm{k}=$ $2, \mathrm{~T}=16$ et $\alpha=0.05$, la probabilité critique (probabilité d'acceptation de l'hypothèse $\mathrm{HO}$ ) de ce test est inférieure à $5 \%$ (prob (F-statistic) $=$ $0.000000<5 \%$ ). Le coefficient de détermination $\left(R^{2}\right)$ corrobore fortement ce bon résultat de l'ajustement global du modèle (son pouvoir explicatif est de $99 \%$ ). Le $t$ de Student (y compris celui du terme constant, C) de DEPPUB a une probabilité critique inférieure à $5 \%$ (prob ( $t$ Statistic) $=0.0000<5 \%)$. Autrement dit, le coefficient de régression de IP

$\mathrm{C}_{91}$ sur DEPPUB est significativement différent de 0 . L'expansion des dépenses publiques liée à celle des recettes d'exportations pétrolières a bien contribué à la hausse du niveau moyen des prix. Le coefficient de corrélation linéaire $\left(R_{\mathrm{IPC} 91, D E P P U B}=\right.$ +0.99 ) corrobore fortement cette relation réciproque positive entre IPC $_{91}$ et DEPPUB. La statistique $d$ de Durbin-Watson $(d=1.77)$ est bonne (elle ne présage pas, à $\alpha=0.05=5 \%$, d'autocorrélation des résidus). Pour $\mathrm{m}=1, \mathrm{~T}=16$ et $\alpha=0.05=5 \%$, il advient des $d_{1}=1.10$ et $d_{2}=1.37$ avec $\mathrm{d}_{2}=1.37<\mathrm{d}=1.77<4-\mathrm{d}_{2}=2.63$.

À son tour, cette élévation des prix internes a provoqué la baisse du prix relatif des biens échangeables et des biens non échangeables, synonyme d'une appréciation du taux de change (effectif) réel du franc CFA $(T C R=R=$ S.P $\left.P_{B E} / P_{B N E}\right)$.

La mise en exergue de cette corrélation (négative) entre l'indice des prix à la consommation et le taux de change effectif réel est faite à partir de ce modèle économétrique (voir les séries chronologiques afférentes aux variables TCER et IPC $C_{91}$ en annexe).

$\mathrm{TCER}_{\mathrm{t}}=\mathrm{aIPC}_{91 \mathrm{t}}+\mathrm{C}+$ Ut tels que TCER $=$ taux de change effectif réel du franc $\mathrm{CFA}=$ variable endogène ou expliquée; IPC $_{91}=$ indice des prix à la consommation (base $100=1$ 991) = variable exogène ou explicative; $\mathrm{a}=$ paramètre exprimant le comportement de TCER suite à une variation de $\mathrm{IPC}_{91} ; \mathrm{C}=$ constante non nulle; $\mathrm{U}_{\mathrm{t}}=$ résidu; $\alpha=$ risque d'erreur $=5 \% ; \mathrm{T}=$ nombre d'observations $=17 ; \mathrm{k}=$ nombre de variables explicatives (terme constant compris $)=2$.
Il ressort, avec un seuil de confiance $(\beta=1-\alpha)=$ seuil de confiance de $95 \%$ et en procédant par tâtonnement quant à l'ordre d'intégration des variables, ce modèle économétrique.

$$
\mathrm{D}\left(\mathrm{D}\left(\text { TCER }_{\mathrm{t}}\right)\right)=-\underset{\left(-3.818^{*}\right)}{-1.284 \mathrm{D}\left(\mathrm{D}\left(\text { IPC91 }_{\mathrm{t}}\right)\right)+}+0.540+\mathrm{e}_{\mathrm{t}}
$$

Ainsi, il existe bien une corrélation négative entre la variable TCER et celle IPC ${ }_{91}$. La hausse du niveau moyen des prix à la consommation a provoqué une baisse du TCER du franc CFA, synonyme de son appréciation réelle. Le coefficient de régression de TCER sur IPC ${ }_{91}$ a bien le signe attendu $(\hat{a}=-1.284$ $<0$ ), signe d'une relation négative entre ces deux variables. La statistique $F$ de Fisher indique que le modèle est bon. Pour $\mathrm{k}=2, \mathrm{~T}=15$ et $\alpha=0.05$, la probabilité critique (probabilité d'acceptation de l'hypothèse H0) de ce test est inférieure à $5 \%$ (prob $(F$-statistic $)=0.00167<5 \%)$. Le coefficient de détermination $\left(R^{2}\right)$ ne corrobore cependant pas vraiment le résultat de l'ajustement global du modèle (son pouvoir explicatif est à peine de $50 \%$ ). Le $t$ de Student de la variable IPC 91 a une probabilité critique inférieure à $5 \%($ prob $(t-$ Statistic $)=0.0017$ $<5 \%$ ). Le coefficient de régression de TCER sur $\mathrm{IPC}_{91}$ est significativement différent de 0 . Autrement dit, ces deux variables sont significativement et négativement corrélées. Le coefficient de corrélation linéaire $\left(R_{T C E R, \mathrm{IPC} 91}=\right.$ +0.7 ) corrobore leur relation réciproque. $\mathrm{La}$ statistique $d$ de Durbin-Watson $(d=1.916)$ est bonne (elle ne présage pas, à $\alpha=0.05=5 \%$, d'autocorrélation des résidus). Pour m (nombre de variables explicatives, terme constant exclu) $=1, \mathrm{~T}$ (nombre d'observations) $=15$ et $\alpha=0.05=5 \%$, il advient des $\mathrm{d}_{1}=1.08$ et $\mathrm{d}_{2}=1.36$ avec $\mathrm{d}_{2}=1.36<\mathrm{d}$ $=1.916<4-\mathrm{d}_{2}=2.64$.

Enfin, la surévaluation réelle du franc CFA (baisse du TCER) a entraîné une détérioration de la compétitivité des produits hors boom exposés au commerce international (cacao, café, bois, mines) et donc, un squeeze (contraction) de leur production.

Afin de faire ressortir la corrélation négative entre l'appréciation réelle du franc CFA et le squeeze de la production de cacao (produit hors boom exposé à la concurrence internationale arbitrairement choisi ici) en raison de la détérioration de sa compétitivité, nous avons procédé à la construction de ce modèle économétrique. 
PRODCACAO $_{\mathrm{t}}=\mathrm{aTCER}_{\mathrm{t}}+\mathrm{C}+\mathrm{Ut}$ tels que $\mathrm{PRODCACAO}=$ production de cacao $=$ variable expliquée ou endogène; TCER $=$ taux de change effectif réel $=$ variable explicative ou exogène; $\mathrm{a}=$ paramètre exprimant le comportement de PRODCACAO suite à une variation de TCER; $\mathrm{C}=$ constante non nulle; $\mathrm{U}=$ résidu; $\alpha=$ risque d'erreur $=5 \% ; \mathrm{T}=$ nombre d'observations $=18 ; \mathrm{k}$ $=$ nombre de variables explicatives (terme constant compris $)=2$.

\section{Enfin, la surévaluation réelle du franc CFA (baisse du TCER) a entrâné une détérioration de la compétitivité des produits hors boom exposés au commerce international (cacao, café, bois, mines).}

Dans ce cadre, nous avons préalablement procédé, à partir des séries temporelles de PRODCACAO et TCER en annexe, au test d'une relation de cointégration entre ces variables avec une estimation éventuelle du modèle à correction d'erreur (MCE). La théorie économétrique, basée sur l'algorithme en deux étapes d'Engle et Granger ${ }^{19}$, exige que les séries temporelles soient intégrées du même ordre (en l'occurrence en I(1)) afin de présumer un risque de cointégration entre elles. Dans le cas contraire, si elles ne sont pas intégrées du même ordre (en I(1)), la procédure est arrêtée.

Pour mener à bien ce test de cointégration, nous avons procédé, dans un premier temps, à l'étude des propriétés des variables endogène et exogène (PRODCACAO et TCER) en termes de stationnarité.

Les résultats du test de Dickey-Fuller ${ }^{20}$ Augmented $(D F A)$ indiquent que ces dernières sont, toutes les deux, intégrées à l'ordre 1 et ce, à $\alpha=0.05=5 \%$.

Tableau 5 - Résultats du test de racine unitaire sur les variables du modèle

\begin{tabular}{|c|c|c|c|c|c|}
\hline Variables & Constante & Trend & Valeur du test & Valeur critique & Conclusion \\
& & & & $\mathbf{5 \%}$ & \\
\hline PRODCACAO & Oui & Non & $\mathbf{- 1 . 2 2 6}$ & $\mathbf{- 3 . 0 5 2}$ & \\
D(PRODCACAO) & Oui & Non & $\mathbf{- 5 . 3 8 8}$ & $\mathbf{- 3 . 0 6 5}$ & I(1) \\
\hline TCER & Oui & Non & $-\mathbf{- 1 . 5 3 3}$ & $\mathbf{- 3 . 0 5 2}$ & \\
D(TCER) & Oui & Non & $-\mathbf{3 . 4 7 9}$ & $\mathbf{- 3 . 0 6 5}$ & I(1) \\
\hline
\end{tabular}

Tableau élaboré à partir des résultats fournis par le logiciel Eviews basic 3.1

Ainsi, PRODCACAO et TCER, étant du même ordre d'intégration, I(1), le risque de cointégration est présumé et la procédure se poursuit avec le test de Johansen ${ }^{21}$. Nous avons procédé à celui-ci sous l'hypothèse de présence d'une ten- dance linéaire dans les données et plus précisément, de présence d'une tendance linéaire dans les séries et d'une constance dans les relations de cointégration.

Tableau 6 - Johansen Cointegration Test

\begin{tabular}{|c|c|c|c|c|}
\hline \multicolumn{5}{|c|}{$\begin{array}{l}\text { Date: } 21 / 06 / 11 \text { Time: } 01: 13 \\
\text { Sample: } 19701987 \\
\text { Included observations: } 17 \\
\text { Test assumption: Linear deterministic trend in the data } \\
\text { Series: PRODCACAO TCER } \\
\text { Lags interval: No lags } \\
\end{array}$} \\
\hline Eigenvalue $\left(\lambda_{\mathrm{i}}\right)$ & $\begin{array}{l}\text { Likelihood } \\
\text { Ratio }\end{array}$ & $\begin{array}{c}5 \text { Percent } \\
\text { Critical Value }\end{array}$ & $\begin{array}{c}1 \text { Percent } \\
\text { Critical Value }\end{array}$ & $\begin{array}{c}\text { Hypothesized } \\
\text { No. of CE(s) }\end{array}$ \\
\hline $\begin{array}{l}0.159685 \\
0.075731\end{array}$ & $\begin{array}{l}4.296431 \\
1.338790\end{array}$ & $\begin{array}{c}15.41 \\
3.76\end{array}$ & $\begin{array}{r}20.04 \\
6.65\end{array}$ & $\begin{array}{c}\text { None } \\
\text { At most } 1\end{array}$ \\
\hline
\end{tabular}

*(**) denotes rejection of the hypothesis at 5\%(1\%) significance level

L.R. rejects any cointegration at $5 \%$ significance level 
$\grave{A}$ partir de la statistique $\lambda_{\text {trace }}=-\mathrm{n} \Sigma \ln \left(1-\lambda_{\mathrm{i}}\right)$, le nombre de relations de cointégration entre PRODCACAO et TCER est recherché par exclusion d'hypothèses ${ }^{22}$ alternatives comme suit.

Premier cas : rang de la matrice M égal à $0(\mathrm{r}=0)$.

$$
\begin{aligned}
\lambda_{\text {trace }}=-\mathrm{n} \Sigma & \ln \left(1-\lambda_{\mathrm{i}}\right)=-17\left[\ln \left(1-\lambda_{1}\right)+\ln \left(1-\lambda_{2}\right)\right] \\
& =-17[\ln (1-0.159)+\ln (1-0.075)] \\
& =-17(-0.1739-0.0787)=4.296 .
\end{aligned}
$$

Or, la valeur critique ( 15.41 pour un seuil à $(=5 \%)$ étant supérieure à la statistique $\lambda_{\text {trace }}$ de Johansen $\left(\lambda_{\text {trace }}=4.296<5 \% C\right.$ - $\left.V=15.41\right), \mathrm{H} 0$ est acceptée. Le rang de la matrice $\mathrm{M}$ étant 0 , il y a donc finalement rejet de l'hypothèse d'une relation de cointégration $(H I)$ entre PRODCACAO et TCER. Autrement dit, il n'existe pas ici de relation de cointégration, car ces variables sont toutes intégrées en niveau (elles sont toutes stationnaires). En conséquence, la modélisation MCE ne peut se poursuivre (elle doit s'arrêter là) et l'utilisation, en régression directe, du modèle économétrique à des fins prévisionnelles ne risque pas de s'avérer désastreuse.

\section{Graphique 11 - Évolution du PRODCACAO et de TCER, 1970-1985}

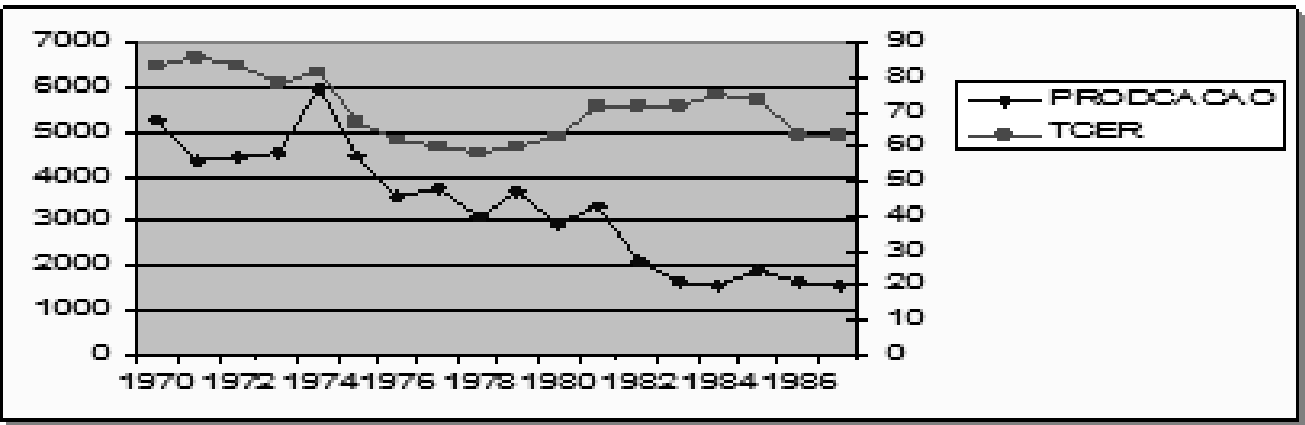

Graphique élaboré à l'aide des données de PRODCACAO et TCER

Dès lors, il ressort, avec un seuil de confiance $(\beta$ $=1-\alpha)=95 \%$ et en procédant par tâtonnement, cette relation de long terme.

$\mathrm{D}\left(\mathrm{D}\left(\right.\right.$ PRODCACAO $\left.\left._{\mathrm{t}}\right)\right)=119.29 \mathrm{D}\left(\mathrm{D}\left(\mathrm{TCER}_{\mathrm{t}}\right)\right)+$ $71.83+\mathrm{e}_{\mathrm{t}}$

$$
\left(4.023^{*}\right)
$$

Ainsi, il existe bien une corrélation positive entre TCER et PRODCACAO. La baisse du taux de change effectif réel du franc CFA (appréciation réelle) liée plus en amont au boom du pétrole a bien provoqué une régression de la production du cacao. Le coefficient de régression de PRODCACAO sur TCER a bien le signe attendu $(\hat{a}=+119.29>0)$, signe d'une relation positive entre ces deux variables. La statistique $F$ de Fisher indique que le modèle est globalement significatif. Pour $\mathrm{k}=2, \mathrm{~T}=16$ et $\alpha=0.05$, la probabilité critique (probabilité d'acceptation de l'hypothèse HO) de ce test est inférieure à $5 \%$ $($ prob $(F$-statistic $)=0.001258<5 \%)$. Le coefficient de détermination $\left(R^{2}\right)$ corrobore très faiblement ce bon résultat de l'ajustement global du modèle (son pouvoir explicatif est seulement de $54 \%$ ). La statistique $t$ de Student de la variable TCER a une probabilité critique inférieure à $5 \%$ $($ prob $(t$-Statistic $)=0.0013<5 \%)$. Le coefficient de régression de PRODCACAO sur TCER est significativement différent de 0 . Autrement dit, ces deux variables sont significativement et positivement corrélées (l'appréciation réelle $d u$ franc CFA a bien contribué à la chute de la production cacaoyère). Le coefficient de corrélation linéaire $\left(R_{P R O D C A C A O, T C E R}=+0.73\right)$ corrobore leur relation réciproque positive. La statistique $d$ de Durbin-Watson $(d=2.279)$ est bonne (elle ne présage pas, à $\alpha=0.05=5 \%$, d'autocorrélation des résidus). Pour m (nombre de variables explicatives, terme constant exclu $)=$ $1, \mathrm{~T}$ (nombre d'observations $)=16$ et $\alpha=0.05=$ $5 \%$, il advient des $\mathrm{d}_{1}=1.10$ et $\mathrm{d}_{2}=1.37$ avec $\mathrm{d}_{2}=$ $1.37<\mathrm{d}=2.279<4-\mathrm{d}_{2}=2.63$.

De la sorte, l'appréciation réelle du franc CFA, induite plus en amont par le boom pétrolier, a provoqué une perte de compétitivité du cacao et partant de là, une baisse relative de la production et des recettes d'exportations de ce produit agricole. 


\section{CONCLUSION}

\section{Après la brusque montée du prix du brut des années 1970, ce pays a connu une \\ transformation de sa base économique qui est aujourd'hui dominée par le pétrole au détriment des activités hors boom tournées vers les marchés intérieurs et internationaux.}

Selon le modèle du «syndrome hollandais », le développement de toute ressource naturelle destinée à l'exportation ou au remplacement des importations conduit, à travers des effets de «déplacement des ressources productives » et de «dépense », à l'appréciation du taux de change (effectif) réel et au déclin relatif de la production domestique des biens exposés au commerce international hors boom. De la sorte, si le secteur pétrolier, grâce à son boom lié en amont à la politique pétrolière de la décennie 1970 , a permis au Gabon de connaître de bons résultats économiques, il ne lui a, par contre, pas permis de conjurer «la malédiction du pétrole » connue sous le nom de «syndrome hollandais ». En effet, après la brusque montée du prix du brut des années 1970, ce pays a connu une transformation de sa base économique qui est aujourd'hui dominée par le pétrole au détriment des activités hors boom tournées vers les marchés intérieurs et internationaux. La vérification de cette théorie du «syndrome hollandais » a fourni d'assez bons résultats du point de vue macroéconomique et économétrique. Le boom du pétrole s'est accompagné d'une régression du niveau relatif des activités traditionnelles (café, cacao, bois, mines...) dans le total du $\mathrm{PIB}$, des recettes d'exportations et budgétaires. $\mathrm{La}$ monétisation constante des recettes d'exportations pétrolières en devises a été, dès le milieu de la décennie 1980, un puissant facteur de déstabilisation de l'économie gabonaise au sens d'accélérer les tensions inflationnistes, de faire apprécier en termes réels le taux de change du franc CFA, de faire baisser la compétitivité-prix internationale des produits non exposés et exposés hors boom et de contribuer à l'atrophie (l'hypertrophie) du secteur hors pétrole (pétrole).

\section{BIBLIOGRAPHIE}

${ }^{1}$ Nowak, J-J. (1994). Le boom du café et du cacao en Côted'Ivoire: une étude de cas de syndrome néerlandais. Revue d'économie du développement, 52-75.
${ }^{2}$ Campan, E. et Grimaud, A. (1989). Le syndrome hollandais. Revue d'économie politique, 6, 810-834.

${ }^{3}$ Daniel, P. (1985). Problèmes d'ajustements consécutifs au mal néerlandais. $O C D E$.

${ }^{4}$ Mace, G. et Pétry, F. (2000). Guide d'élaboration d'un projet de recherche. Québec, Québec: Presses de l'Université Laval (PUL), p. 25-31.

${ }^{5}$ Fortin, M.-F., Côté, J. et Fillion, F. (2006). Fondements et étapes du processus de recherche. Montréal, Québec : Chenelière Éducation, chapitre 10, p. 174-177.

${ }^{6}$ Crête, J. et Imbeau, L.M. (1994). Comprendre et communiquer la science. Québec, Québec: Presses de l'Université Laval.

${ }^{7}$ Gregory, R.G. (1976). Some implications of the growth mineral sector. Australian Journal of the Agricultural Economics, 20(2).

${ }^{8}$ Corden, W.M. et Neary, J.P. (1982). Booming sector and desindustrialization in a small open economy. Economic Journal, 92(368), 825-848.

${ }^{9}$ Stolper, W.F. et Samuelson, P.A. (1941). Protection and Real Wages. Review of Economic Studies, 9(1), 58-73.

Ce théorème dit que la hausse du prix d'un bien ou service engendre la hausse (baisse) du prix ou de la rémunération du facteur de production relativement intensif (non-intensif) en ce dernier.

${ }^{10}$ Okoué Edou, J.J.R. (2007). L'économie gabonaise souffre du syndrome hollandais, dit-on! Paris, France : Les Éditions Persée.

${ }^{11}$ Fortin, M.-F. (1996). Le processus de recherche : de la conception à la réalisation. Ville Mont-Royal, Québec : Decarie éditeur, p. 89-98.

${ }^{12}$ Gaulme, F. (1983). Atouts et faiblesses de l'économie gabonaise. Problèmes économiques, (1829), 17.

${ }^{13}$ Okoué Edou, J.J.R. (2007). Pourquoi la dévaluation du franc CFA était-elle vouée à l'échec au Gabon?, Paris, France : L'Harmattan, p. 166-169.

${ }^{14}$ Wunder, S. (2003). Quand le syndrome hollandais rencontre la French Connection: pétrole, macroéconomie et forêts au Gabon. Bogor, Indonésie : CIFOR, p. 22-24.

${ }^{15}$ Ellman, M. (1981). Natural gas, restructuring and deindustrialisation: The Dutch experience of industrial policy, B. a. Brailovsky.

${ }^{16}$ Scherr, S. J. (1989). Agriculture in an export boom economy: a comparative analysis of policy and performance in Indonesia, Mexico and Nigeria. World Development, 17(4) 543-560.

${ }^{17}$ Bourbonnais, R. (2002). Économétrie, manuel et exercices corrigés (4e éd.). Malakoff, France : Dunod.

${ }^{18}$ Okoué Edou, J.J.R. (2007). Op. cit. Paris, France : L'Harmattan, p. 166-169.

${ }^{19}$ Engle, R.E. et Granger, C.W.J. (1951). Cointegration and Error-correction: representation, estimation and testing. Econometrica, 38. 
${ }^{20}$ Dickey, D. et Fuller, W. (1979). Distribution of the estimators for autoregressive time series with unit root. Journal of the American Statistical Association, 74(366). ${ }^{21}$ Johansen, S. (1988). Statistical analysis of cointegration vectors. Journal of Economic Dynamics and Control, 12.

${ }^{22}$ Johansen, S. et Juselius, K. (1990). Maximum likelihood estimation and to the demand for money inference on cointegration with application. Oxford Bulletin of Economics and Statistics, 52(2). 


\section{ANNEXES}

1. Séries temporelles des variables MOA et VAP

\begin{tabular}{||c||c|c|}
\hline \hline Observations & MOA (en unités indiquées) & VAP (en Mds francs CFA) \\
\hline \hline 1986 & $\mathbf{2 . 3 0 0}$ & $\mathbf{2 2 5 , 1}$ \\
1987 & 2.271 & $\mathbf{2 4 6 , 9}$ \\
1988 & $\mathbf{2 . 2 7 9}$ & $\mathbf{1 6 8 , 6}$ \\
1989 & 2.181 & $\mathbf{3 3 4 , 2}$ \\
1990 & 2.260 & $\mathbf{4 8 4 , 6}$ \\
1991 & 2.290 & $\mathbf{4 5 5 , 8}$ \\
1992 & 2.412 & $\mathbf{3 9 4 , 6}$ \\
1993 & 2.175 & $\mathbf{4 0 3 , 4}$ \\
1994 & 2.197 & $\mathbf{9 1 1 , 2}$ \\
1995 & 2.216 & $\mathbf{9 6 9 , 8}$ \\
1996 & 2.090 & $\mathbf{1 . 2 7 8 , 7}$ \\
1997 & 2.075 & $\mathbf{1 . 2 7 4 , 4}$ \\
1998 & 2.162 & $\mathbf{7 1 3 , 8}$ \\
1999 & 2.065 & $\mathbf{1 . 0 4 9 , 4}$ \\
2000 & $\mathbf{2 . 0 6 6}$ & $\mathbf{1 . 7 0 2 , 0}$ \\
\hline
\end{tabular}

Sources : BEAC, DGE - TBE.

2. Séries temporelles des variables DEPPUB et REXPET

\begin{tabular}{||c||c||c||}
\hline \hline Observations & DEPPUB (en $M d s$ francs $C F A$ ) & REXPET (en $M d s$ francs $C F A$ ) \\
\hline \hline 1982 & $\mathbf{4 5 3 , 5}$ & $\mathbf{4 9 3 , 8}$ \\
1983 & $\mathbf{5 6 2 , 0}$ & $\mathbf{6 0 0 , 1}$ \\
1984 & $\mathbf{5 9 7 , 0}$ & $\mathbf{7 3 5 , 7}$ \\
1985 & $\mathbf{6 7 9 , 0}$ & $\mathbf{7 3 1 , 8}$ \\
1986 & $\mathbf{6 6 8 , 2}$ & $\mathbf{2 4 4 , 6}$ \\
1987 & $\mathbf{3 6 9 , 2}$ & $\mathbf{2 6 6 , 3}$ \\
1988 & 333,7 & $\mathbf{2 2 2 , 6}$ \\
1989 & $\mathbf{3 4 7 , 2}$ & $\mathbf{3 7 6 , 8}$ \\
1990 & 387,9 & $\mathbf{5 4 1 , 1}$ \\
1991 & 398,3 & $\mathbf{5 1 6 , 0}$ \\
1992 & $\mathbf{4 7 9 , 7}$ & $\mathbf{4 9 7 , 7}$ \\
1993 & $\mathbf{4 3 2 , 8}$ & $\mathbf{4 9 5 , 1}$ \\
1994 & $\mathbf{5 9 2 , 3}$ & $\mathbf{1 . 0 1 9 , 2}$ \\
1995 & $\mathbf{6 5 3 , 8}$ & $\mathbf{1 . 0 2 7 , 0}$ \\
1996 & $\mathbf{8 2 8 , 6}$ & $\mathbf{1 . 3 3 5 , 1}$ \\
1997 & $\mathbf{9 8 0 , 2}$ & $\mathbf{1 . 3 6 9 , 7}$ \\
\hline
\end{tabular}

Sources : BEAC, DGE - TBE.

3. Séries temporelles des variables DEPPUB et IPC 91

\begin{tabular}{||c||c||c|}
\hline Observations & DEPPUB $($ en $M d s$ francs $C F A)$ & IPC $_{91}$ (en unités indiquées $)$ \\
\hline \hline 1970 & $\mathbf{2 0 , 0 0}$ & $\mathbf{1 9 , 1 0}$ \\
1971 & $\mathbf{2 4 , 5 2}$ & $\mathbf{1 9 , 8 0}$ \\
1972 & $\mathbf{3 1 , 0 0}$ & $\mathbf{2 0 , 5 0}$ \\
1973 & $\mathbf{3 6 , 9 4}$ & $\mathbf{2 1 , 9 0}$ \\
1974 & $\mathbf{4 8 , 7 1}$ & $\mathbf{2 4 , 5 0}$ \\
1975 & $\mathbf{1 2 0 , 9 1}$ & $\mathbf{3 1 , 5 0}$ \\
1976 & 193,11 & $\mathbf{3 5 , 4 0}$ \\
1977 & 255,79 & $\mathbf{4 2 , 2 0}$ \\
1978 & $\mathbf{2 4 2 , 4 5}$ & $\mathbf{4 6 , 8 0}$ \\
1979 & $\mathbf{2 8 2 , 4 2}$ & $\mathbf{5 0 , 5 0}$ \\
1980 & $\mathbf{3 1 3 , 7 1}$ & $\mathbf{5 6 , 8 0}$ \\
1981 & $\mathbf{4 0 4 , 5 0}$ & $\mathbf{6 1 , 7 0}$ \\
1982 & $\mathbf{4 5 3 , 5 0}$ & $\mathbf{7 2 , 0 0}$ \\
1983 & $\mathbf{5 6 2 , 0 0}$ & $\mathbf{8 4 , 5 0}$ \\
1984 & $\mathbf{5 9 7 , 0 0}$ & $\mathbf{9 0 , 4 0}$ \\
1985 & $\mathbf{6 7 9 , 0 0}$ &
\end{tabular}

Sources : BEAC, DGE - TBE. 
4. Séries temporelles des variables IPC ${ }_{91}$ et TCER

\begin{tabular}{||c||c|c||}
\hline Années & $\begin{array}{c}\text { IPC91 } \\
\text { (en unités indiquées) }\end{array}$ & $\begin{array}{c}\text { TCER } \\
\text { (en unités indiquées) }\end{array}$ \\
\hline \hline 1975 & $\mathbf{3 1 , 5}$ & $\mathbf{6 7 , 3}$ \\
1976 & $\mathbf{3 5 , 4}$ & $\mathbf{6 1 , 9}$ \\
1977 & $\mathbf{4 2 , 2}$ & $\mathbf{6 0 , 2}$ \\
1978 & $\mathbf{4 6 , 8}$ & $\mathbf{5 8 , 1}$ \\
1979 & $\mathbf{5 0 , 5}$ & $\mathbf{6 0 , 0}$ \\
1980 & $\mathbf{5 6 , 8}$ & $\mathbf{6 3 , 1}$ \\
1981 & $\mathbf{6 1 , 7}$ & $\mathbf{7 1 , 8}$ \\
1982 & $\mathbf{7 2 , 0}$ & $\mathbf{7 1 , 7}$ \\
1983 & $\mathbf{7 9 , 5}$ & $\mathbf{7 1 , 8}$ \\
1984 & $\mathbf{8 4 , 1}$ & $\mathbf{7 4 , 9}$ \\
1985 & $\mathbf{9 0 , 4}$ & $\mathbf{7 3 , 8}$ \\
1986 & $\mathbf{9 6 , 0}$ & $\mathbf{6 3 , 4}$ \\
1987 & $\mathbf{9 5 , 0}$ & $\mathbf{6 3 , 3}$ \\
1988 & $\mathbf{8 6 , 8}$ & $\mathbf{7 3 , 8}$ \\
1989 & $\mathbf{9 2 , 9}$ & $\mathbf{7 3 , 5}$ \\
1990 & $\mathbf{9 8 , 5}$ & $\mathbf{6 7 , 7}$ \\
1991 & $\mathbf{1 0 0 , 0 0}$ & $\mathbf{8 1 , 7}$ \\
\hline
\end{tabular}

Sources : BEAC, DGE - TBE.

5. Séries temporelles de TCER et PRODCACAO

\begin{tabular}{||c||c||c||}
\hline Années & $\begin{array}{c}\text { PRODCACA0 } \\
\text { (en Tonnes) }\end{array}$ & $\begin{array}{c}\text { TCER } \\
\text { (en unités indiquées) }\end{array}$ \\
\hline \hline 1970 & $\mathbf{5 . 2 7 1}$ & $\mathbf{8 3 , 4}$ \\
1971 & 4.342 & $\mathbf{8 5 , 6}$ \\
1972 & $\mathbf{4 . 4 4 2}$ & $\mathbf{8 3 , 4}$ \\
1973 & 4.522 & $\mathbf{7 8 , 2}$ \\
1974 & $\mathbf{6 . 0 0 0}$ & $\mathbf{8 1 , 6}$ \\
1975 & 4.470 & $\mathbf{6 7 , 3}$ \\
1976 & 3.570 & $\mathbf{6 1 , 9}$ \\
1977 & 3.720 & $\mathbf{6 0 , 2}$ \\
1978 & 3.095 & $\mathbf{5 8 , 1}$ \\
1979 & 3.668 & $\mathbf{6 0 , 0}$ \\
1980 & 2.935 & $\mathbf{6 3 , 1}$ \\
1981 & 3.353 & $\mathbf{7 1 , 8}$ \\
1982 & 2.095 & $\mathbf{7 1 , 7}$ \\
1983 & 1.647 & $\mathbf{7 1 , 8}$ \\
1984 & 1.569 & $\mathbf{7 4 , 9}$ \\
1985 & 1.872 & $\mathbf{7 3 , 8}$ \\
1986 & 1.628 & $\mathbf{6 3 , 4}$ \\
1987 & 1.574 & $\mathbf{6 3 , 3}$ \\
\hline
\end{tabular}

Sources : BEAC, DGE - TBE. 